\title{
A study on surveillance video abstraction techniques
}

\begin{abstract}
The goal of surveillance video abstraction is to generate a video abstract that includes important events and object by eliminating the redundant frames, lacking from activity in original video. Although many research and progresses have been done in video abstraction, the developed approaches either fail to accurately and effectively cover the overall visual content of video or they are computationally expensive in term of time or process. In this paper, firstly we critically review the applicable video abstraction techniques in surveillance domain based on our hierarchical classification, and then briefly introduce a new approach for generating a static surveillance video abstraction, which mitigate the drawbacks of reviewed approaches.
\end{abstract}

Keyword: Video abstraction; Keyframe extraction; Surveillance video abstraction; Video skimming; Video summary 